\title{
Cigarette smoke stimulates the stemness of renal cancer stem cells via Sonic Hedgehog pathway
}

\author{
Weiwei Qian ${ }^{1}$, Xiaochuan Kong ${ }^{1}$, Tao Zhang ${ }^{1}$, Dengdian Wang ${ }^{1}$, Jin Song ${ }^{1}$, Yuan Li ${ }^{2}$, Xiaoting Li ${ }^{2}$, Hao Geng ${ }^{1}$, Jie Min ,
} Qi Kong ${ }^{3}$, Jie Liu', Zhiqi Liu', Daming Wang' ${ }^{1}$, Zhiqiang Zhang ${ }^{1}$, Dexin $\mathrm{Yu}^{1}$ and Caiyun Zhong ${ }^{2}$

\begin{abstract}
Cancer stem cells (CSCs) are essentially responsible for tumor initiation, growth, progression, metastasis and recurrence, and cigarette smoke (CS) is closely involved in the occurrence and development of kidney cancer. However, the effect of CS on renal CSCs has not been elucidated yet. In the present study, tumorsphere formation assay was used to enrich renal CSCs from 786-O and ACHN cells. We illustrated that CS effectively promoted renal CSCs stemness by enhancing tumorsphere formation, increasing the expression of renal CSCS markers (CD133, CD44, ALDHA1, Oct4, and Nanog) and elevating $\mathrm{CD}_{133^{+}}$cell population. Moreover, our results showed that CS triggered the activation of Sonic Hedgehog (SHH) pathway, while inhibition of SHH pathway dampened the promotive effects of CS on renal CSCS. Finally, higher levels of renal CSCS markers and SHH pathway-related proteins were observed in kidney cancer tissues from smokers than non-smoking cancer tissues. Taken together, these results demonstrated the important role of SHH pathway in regulating CS-induced renal CSCs stemness augment. Findings from this study could provide new insight into the molecular mechanisms of CS-elicited stemness of renal CSCs.
\end{abstract}

\section{Introduction}

Among urologic tumors, renal cell carcinoma (RCC) is characterized as the highest cancer-specific mortality rate, and the 5-year survival rate for patients with metastatic disease is only $12 \%{ }^{1}$. High metastatic index and resistance to radiation and chemotherapy of RCC are responsible for unpredictable presentation and poor clinical outcome ${ }^{2}$. Therefore, discovery of novel approaches for the treatment of RCC is urgent.

Cancer stem cells (CSCs), a small subpopulation of cancer cells, are critically implicated in tumor occurrence, growth, progression, metastasis, therapy resistance,

Correspondence: Dexin Yu (ay2fy_uro@126.com) or

Caiyun Zhong (cyzhong@njmu.edu.cn)

'Department of Urology, The Second Affiliated Hospital of Anhui Medical University, Hefei 230032, China

${ }^{2}$ Department of Nutrition and Food Safety, School of Public Health, Nanjing Medical University, Nanjing 211166, China

Full list of author information is available at the end of the article

These authors contributed equally: Weiwei Qian and Xiaochuan Kong relapse, and poor prognosis ${ }^{3,4}$. CSCs possess several distinct features including clonogenic ability, self-renewal, expression of stem cell markers, growth in non-adhesive spheroids and multipotency capacity ${ }^{5-7}$. CSCs have been identified and isolated from numerous solid malignancies including $\mathrm{RCC}^{8-10}$. Herein, a better understanding of the molecular mechanisms of CSCs is necessary to overcome the current treatment limitations.

Sonic Hedgehog (SHH) signaling pathway has emerged as a critical component of CSCs. Aberration activation of SHH pathway has been implicated in the initiation and progression of multiple cancer types ${ }^{11}$. The activation of SHH protein relies on its binding to its receptor Patched (PTCH), which initiates a downstream signaling cascade, ultimately regulating the target genes including CD133, CD44, and Nanog ${ }^{12}$. In the absence of SHH, PTCH suppresses the transmembrane protein Smoothened (Smo) activity, which then represses Smo to activate an intracellular signal transduction cascade through Gli 
transcription factors ${ }^{13,14}$. There are three Gli transcription factors: Gli1 functions as a transcription activator, Gli2 and Gli3 can act as either repressor or activator, in a context-dependent manner ${ }^{15}$.

A large amount of epidemiological studies have demonstrated that cigarette smoke (CS) is a major established risk factor of $\mathrm{RCC}^{16}$. CS exposure increases the proportion of cancer stem-like cells in lung cancer cells and head and neck cancer cells ${ }^{17}$. To date, however, the underlying molecular mechanisms of CS on kidney CSCs stemness remain to be elucidated.

Therefore, the present study was designed to investigate whether SHH pathway is involved in CS-promoted stemness of kidney CSCs. These novel findings may open new avenues in search of potential interventional target of CS-associated RCC.

\section{Results}

Enrichment of renal CSCs by serum-free medium (SFM) culture

CSCs have the capacity to form three-dimensional structures or spheres, when cultured with SFM. Tumoresphere formation assay via SFM is widely used in isolation and enrichment of CSCs in vitro. To evaluate the characteristic of renal CSCs, we cultured two human RCC cell lines 786-O and ACHN under the conditions of SFM and serum-supplied medium (SSM), respectively. As shown in Fig. 1a, 786-O and ACHN cells grew adherently in SSM; under SFM, cells were able to form threedimensional tumorspheres. Since renal CSCs express CSCs markers including CD133, CD44, ALDHA1, Oct4, and Nanog, their expression levels were determined in sphere-forming cells as well as in adherent cells. It was revealed that both protein and mRNA expression levels of the above indicated genes were markedly up-regulated in 786-O and ACHN tumorspheres cultured in SFM for 5 days (Figs. 1b, c). Moreover, flow cytometry analysis showed that higher percentage of CD133-positive cells was observed in those sphere-forming cells compared with adherent cells (Fig. 1d). Thus, these results suggested the characteristics of renal CSCs in 786-O and ACHN sphere-forming cells.

\section{CS promotes the stemness of renal CSCs}

To determine the effect of CS extract (CSE) on the viability of the cells, 786-O and ACHN tumorspheres were treated with various concentrations of CSE for 5 days and cell viability was examined by CCK- 8 assay. We found that a significant increase in cell viability was observed in 786-O cells at CSE concentrations of 0.05 and $0.1 \%$, and in ACHN cells at CSE concentrations of 0.05, 0.1 , and $0.25 \%$; cell viability was significantly decreased at 1\% CSE (Fig. 2a). Subsequently, the effect of CS on renal CSCs stemness was investigated. After treatment with
CSE at 0.05 and $0.1 \%$, CSE effectively promoted the size and numbers of 786-O and ACHN tumorspheres (Figs. $2 \mathrm{~b}, \mathrm{c})$. Simultaneously, flow cytometry analysis showed that CSE increased the percentage of CD133-positive cells in those sphere-forming cells (Fig. 2d). Moreover, protein and mRNA expression levels of renal CSCs markers were significantly upregulated by CSE treatment (Figs. 2e, f). In addition, cell proliferation associated proteins (PCNA and Cyclin D1) were markedly increased by CSE (2G). Immunofluorescent staining also revealed that CSE enhanced the expression of $\mathrm{CD} 44$ protein in 786-O and ACHN sphere-forming cells (Fig. 2h). Collectively, these data suggested that CSE promoted the stemness of renal CSCs.

\section{Upregulation of SHH pathway mediates the promotive effects of CS on renal CSCs}

In order to determine whether $\mathrm{SHH}$ pathway was involved in CS-induced stemness of renal CSCs, we next examined the activation of SHH pathway in CSE-treated sphere-forming cells. As shown in Fig. 3, CSE significantly increased the expression of Shh, Smo, Gli1, and Gli2 in both 786-O and ACHN tumorspheres, indicating the activation of SHH pathway in renal CSCs.

To further examine the role of SHH pathway in the stimulative effect of CS on renal CSCs, Vismodegib, an inhibitor of Smo, was used to suppress SHH pathway. Our data showed that Vismodegib treatment inhibited the expression of SHH pathway-related proteins (Fig. 4a). It also showed that Vismodegib treatment resulted in lower tumorsphere formation (Figs. 4b, c) and reduced expression of renal CSCs markers in 786-O and ACHN sphereforming cells (Fig. 4d). Meanwhile, we revealed that the stimulative effects of CS on SHH pathway, tumorsphere formation and renal CSCs markers were abrogated by Vismodegib treatment (Figs. 4a-d). Taken together, these data indicated that CS induced the stemness of renal CSCs through SHH pathway activation.

\section{Alteration of renal CSCs correlates with the smoking status of renal cell carcinoma patients}

The clinical characteristics of a total of 20 renal cell carcinoma patients were descripted in detail in Table 1 . Postoperative tumor tissues were collected for western blotting and immunohistochemistry (IHC) staining to compare the expression of renal CSCs markers in smoker and non-smoker patients. Western blotting showed that higher expression levels of CD133, ALDHA1 and Nanog were observed in smoker tumor tissues than in nonsmoker tumor tissues (Figs. 5a, b). In addition, these western blotting results of CSCs markers were further confirmed by IHC staining (Figs. 5c, d). Moreover, the expression levels of Shh, Smo and Gli1 were also notably increased in smoker tumor tissues (Figs. 5e, f). 


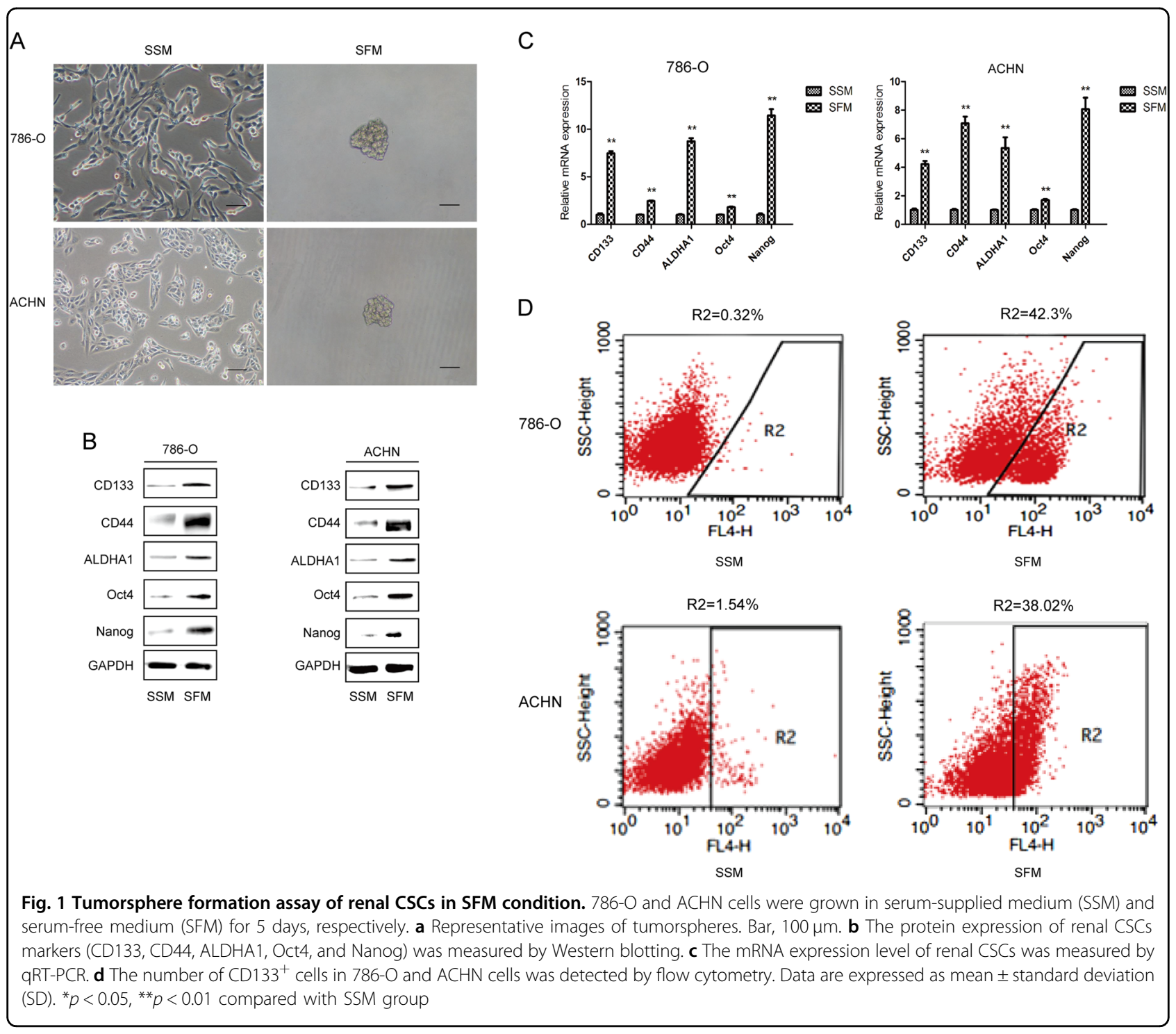

\section{Discussion}

CSCs play a crucial role in the progress, metastasis and recurrence of human cancers. CS is a major risk factor of RCC. SHH pathway is critically implicated in tumorigenic process. However, the molecular mechanisms for the effects of CS on renal CSCs remain unclear. In the present study, we illustrated the pivotal role of SHH pathway in CS-induced enhancement of renal CSCs stemness. These findings could provide new insights into the molecular mechanism of kidney cancer occurrence and advancement.

It has been established that CSCs are able to form stable three-dimensional spheres in vitro. Hence, tumorsphere formation assay in SFM culture condition offers convenient approach for the enrichment and isolation of $\mathrm{CSCs}^{18-20}$. CSCs are characterized by the expression of distinct cell markers. CD133, CD44, ALDHA1, Oct4, and Nanog are widely used for identifying renal CSCs. CD133 has been used to isolate and identify a variety of CSCs including renal $\mathrm{CSCs}^{21}$. The expression of CD133 relates to the prognosis in renal cell carcinoma ${ }^{22}$. CD44 is also a clear renal CSC marker. There is a positive correlation between CD44 expression and tumor metastasis ${ }^{23}$. Besides, ALDHA1 positive cells present higher tumorigenicity than ALDHA1 negative counterparts ${ }^{24}$. Aberrant expression of Oct4 and Nanog, pluripotent stem cell markers of embryo and embryonic stem cells, participates in pluripotent initiation and differentiation ${ }^{25}$. In this study, we showed the tumorsphere formation capacity of both 786-O and ACHN cells cultured in SFM, and markedly increased expression levels of CD133, CD44, ALDH1A1, Oct4, and Nanog in sphere-forming cells. Meanwhile, flow cytometry analysis for the detection of CD133 positive cells was in line with the tumorsphere formation assay and Western blotting data. These results 


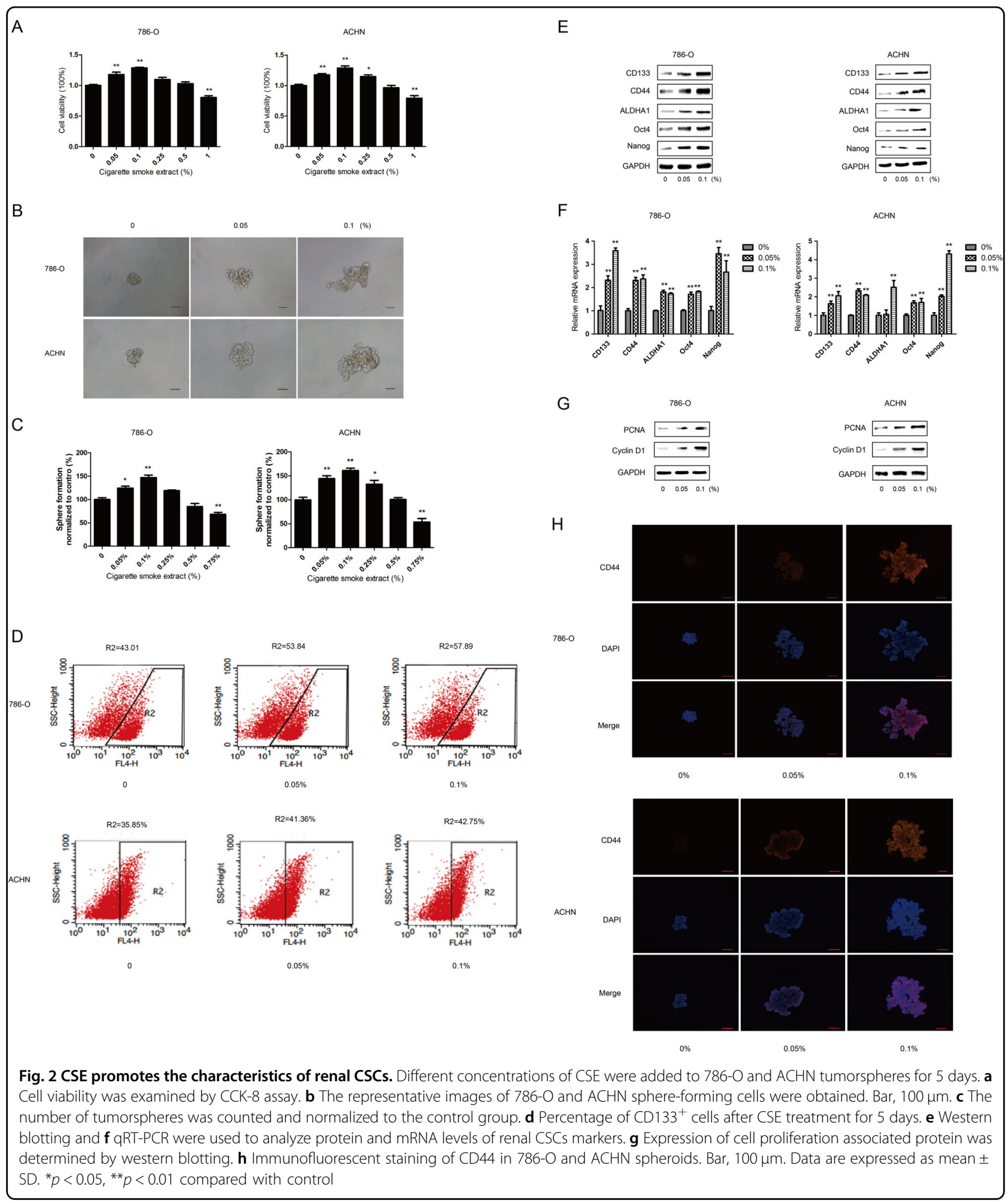

suggested the characteristics of CSCs in these renal cancer cells.

CS is widely considered as a significant and independent risk factor for RCC. In comparison with non-smokers, the risk for RCC has been increased about $50 \%$ in male and $20 \%$ in female smokers ${ }^{26}$. Besides, CS negatively impacts the overall and cancer-specific survival of renal cell carcinoma $^{27}$. What is more, CS is closely associated with 
Table 1 Clinical characteristics of patients $(n=20)$

\begin{tabular}{|c|c|}
\hline Parameters & Number of cases \\
\hline \multicolumn{2}{|l|}{ Gender } \\
\hline Male & $12(60 \%)$ \\
\hline Female & $8(40 \%)$ \\
\hline \multicolumn{2}{|l|}{ Age } \\
\hline$\leqq 50$ & $3(15 \%)$ \\
\hline$>50-\leqq 70$ & 15 (75\%) \\
\hline$>70$ & $2(10 \%)$ \\
\hline \multicolumn{2}{|c|}{ Tumor size $(\mathrm{cm})$} \\
\hline$\leqq 4$ & $11(55 \%)$ \\
\hline$>4-\leqq 7$ & 7 (35\%) \\
\hline$>7$ & $2(10 \%)$ \\
\hline \multicolumn{2}{|l|}{ Tumor stage } \\
\hline pT1a & $11(55 \%)$ \\
\hline pT1b & $6(30 \%)$ \\
\hline рT2a & $1(5 \%)$ \\
\hline pT2b & $1(5 \%)$ \\
\hline pT3 & $1(5 \%)$ \\
\hline \multicolumn{2}{|l|}{ TNM stage } \\
\hline । & 17 (85\%) \\
\hline$\|$ & $2(10 \%)$ \\
\hline III & $1(5 \%)$ \\
\hline IV & $0(0 \%)$ \\
\hline
\end{tabular}

CSCs. CS induces the acquisition of CSCs properties in 40-passage transformed human bronchial epithelial cell ${ }^{28}$. However, no study has addressed the possible relationship between CS and renal CSCs so far. In the present study, we demonstrated that CS significantly enhanced renal CSCs stemness by increasing tumorsphere formation, upregulating the expression levels of renal CSCs markers (CD133, CD44, ALDH1A1, Oct4, and Nanog) and elevating the population of CD133 positive cells. Meanwhile, higher expression of renal CSCs markers was also revealed in RCC tumor tissues of smokers. Together, these data suggested that CS increased the stemness of renal CSCs.

Several embryonic signal pathways, such as $\mathrm{SHH}$, Notch and Wnt/ $\beta$-catenin pathways, have been implicated in CSCs. SHH signaling pathway is essential for the maintenance of $\mathrm{CSCs}^{29}$. In anaplastic thyroid cancer, $\mathrm{SHH}$ pathway maintains the self-renewal property of $\mathrm{CSCs}^{30}$. Inhibition of $\mathrm{SHH}$ pathway blocks pancreatic CSCs growth in vitro and in vivo ${ }^{31}$. In addition, it has been reported that $\mathrm{SHH}$ pathway closely correlates with kidney cancer. Activation of $\mathrm{SHH}$ pathway facilitates renal tumor growth $^{31}$. By targeting SHH pathway, Vitamin D3 triggers its antitumor activity in renal cell carcinoma ${ }^{32}$. We showed in our study that CS induced the activation of $\mathrm{SHH}$ pathway in renal CSCs, as evidenced by increased expression levels of Shh, Smo, Gli1, and Gli2. Furthermore, we demonstrated that the promotive effects of CS on $\mathrm{SHH}$ pathway activation, tumorsphere formation, and renal CSCs markers expression were diminished by Vismodegib-elicited downregulation of $\mathrm{SHH}$ pathway. It is noteworthy that Vismodegib failed to totally rescue the effect of CS on renal CSCs, suggesting that CS-derived cancer stemness could also be driven by other signaling pathways. Liang et al reported that Wnt/ $\beta$-catenin pathway modulates CS-triggered stemness in bladder tumor ${ }^{33}$. Besides, Naoya et al also revealed that CS-specific nitrosamine 4-(methylnitrosamino)-1-(3-pyridyl)-1-butanone increases lung cancer stem cell proportion through Wnt pathway ${ }^{34}$. These findings support our results. Finally, we showed that significantly increased expression levels of $\mathrm{SHH}$ pathway proteins as well as renal CSCs markers were observed in smoker tumor tissues than non-smoker tumor tissues. Taken together, these data revealed that the stimulative effect of CS on renal CSCs stemness was at least partially through the activation of $\mathrm{SHH}$ signaling pathway. In summary, the present study illustrated the important role of $\mathrm{SHH}$ pathway in CS-triggered enhancement of renal CSCs stemness. These findings may provide new insights into the molecular mechanism of CS-associated renal malignancy tumorigenesis and advancement.

\section{Materials and methods}

\section{Cell culture and reagents}

Human kidney cancer cell lines 786-O and ACHN were purchased from Chinese Academy of Typical Culture Collection Cell Bank (Shanghai, China), cultured in $37^{\circ} \mathrm{C}$, $5 \% \mathrm{CO}_{2}$, and saturated humidity. 786-O cells were maintained in RPMI 1640 (Gibco, Carlsbad, CA, USA) medium supplemented with $10 \%$ fetal bovine serum (FBS) and $1 \%$ penicillin/streptomycin. ACHN cells grew in the same condition except in MEM (Gibco, Carlsbad, CA, USA) medium. Vismodegib was acquired from Medchem Express (NJ, USA). Epidermal growth factor (EGF) and basic fibroblast growth factor (bFGF) were acquired from Peprotech (Rocky Hill, NJ, USA). Insulin and 2\% B27 were acquired from Gibco. Primary antibodies including CD133, CD44, ALDHA1, Oct4, Nanog, PCNA, CyclinD1, Shh, Smo, Gli1, Gli2, and GAPDH were purchased from Proteintech (Rocky Hill, NJ, USA). Anti-rabbit and antimouse second antibodies were purchased from ZSGBBIO (Beijing, China). PCR primers of CD133, CD44, 


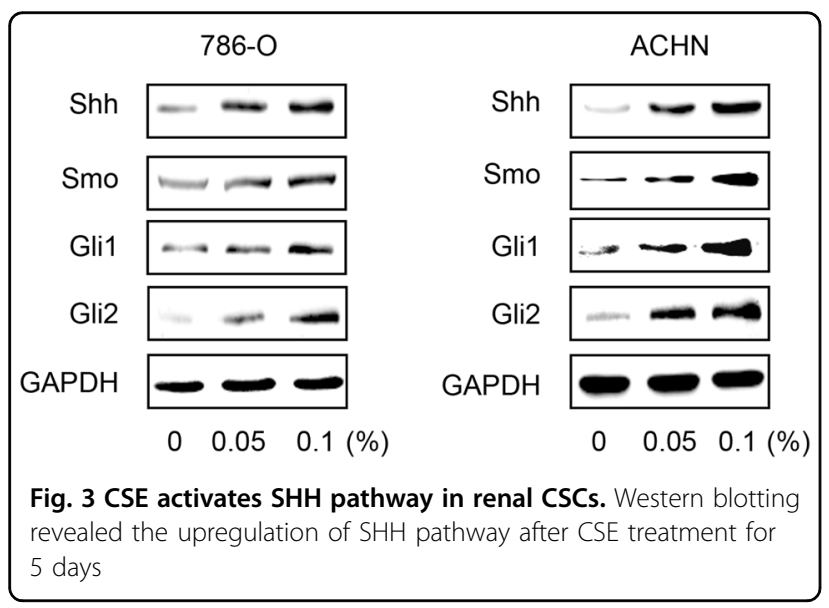

ALDHA1, Oct4, and Nanog were synthesized by Beijing Genomics Institute (Beijing, China).

\section{Tumorsphere formation assay}

786-O and ACHN cells were seeded into 24-well culture plates at 5000 cells per well and cultivated in SFM [Dulbecco's Modified Eagle's Medium: Nutrient Mixture F-12 (DMEM/F12) (Gibco)] supplemented with $20 \mathrm{ng} / \mathrm{mL}$ EGF, $20 \mathrm{ng} / \mathrm{mL}$ bFGF, $5 \mu \mathrm{g} / \mathrm{ml}$ insulin and $2 \%$ B27, and half SFM with additions were renewed every other day. Tumorspheres were visualized by a microscope subsequently (Nikon, Japan).

To investigate the effect of CSE on renal CSCs stemness alterations, various concentrations of CSE were added to each well. After 5 consecutive days of treatment, the number and size of sphere-forming cells were obtained (only sphere diameter $>50 \mu \mathrm{m}$ was counted).

\section{Preparation of CS extract}

CSE was daily prepared for each experiment immediately before use by combusting a filterless 3R4F reference cigarette (University of Kentucky, Kentucky, USA; each cigarette containing $9 \mathrm{mg}$ tar and $0.8 \mathrm{mg}$ nicotine) according to the reported method. After a cigarette was smoked by a vacuum, mainstream smoke was drawn through $10 \mathrm{ml}$ pre-warmed $\left(37^{\circ} \mathrm{C}\right)$ FBS-free DMEM-F12 at the rate of $5 \mathrm{~min} /$ cigarette. Then the CSE stock solution was adjusted to $\mathrm{pH} 7.4$ and then passed through a 0.22 $\mu \mathrm{m}$-pore filter. The obtained mixture was defined as concentration of $100 \%$ CSE. Both 786-O and ACHN cells were treated with desired concentrations of CSE for 5 days. An unlit cigarette was performed with control solution following the same protocol.

\section{Western blotting analysis}

Tumorspheres were collected, washed with ice-cold phosphated-buffer saline (PBS) and then solubilized in RIPA buffer (Thermo Scientific, USA) containing protease inhibitors. Protein concentration was measured by BCA
Protein Assay Kit (Pierce, Rockforsd, WI, USA), and was then subjected to sodium dodecyl sulfate-polyacrylamide gel electrophoresis (SDS-PAGE) and transferred to polyvinylidene difluoride membranes (Millipore, Billerica, USA). After blocking with $5 \%$ defatted milk at room temperature, the transferred membranes were incubated with specific primary antibodies (1:500-1000 dilution) at $4{ }^{\circ} \mathrm{C}$ overnight and then followed by incubating with secondary antibodies. GAPDH was used as a loading control.

\section{Quantitative real-time polymerase chain reaction}

Total RNA was extracted by TRIzol reagent (Invitrogen, Carlsbad, CA, USA) and RNA $(1 \mu \mathrm{g})$ was reversetranscribed into cDNA following the manufacturer's instructions (abm, Canada).

The quantitative real-time PCR (qRT-PCR) was carried out by the Power SYBR Green Master Mix (Applied Biosystems, Foster City, CA, USA) and a LC96 real-time PCR detection system (Roche, Biosystems). Standardized by GAPDH, the mRNA expression levels of CD133, CD44, ALDH1A1, Oct4 and Nanog were measured. Fold changes of gene expression were obtained by the formula $2^{-\Delta \Delta \mathrm{Ct}}$ using comparative threshold cycle $(\mathrm{Ct})$. Primer sequences are listed below:

CD133-F, 5'-TACAACGCCAAACCACGACTGT-3'; CD133-R, 5'-TCTGAACCAATGGAATTCAAGACCCTTT-3'; CD44-F, 5'-GACACATATTGTTTCAATGCTTCAGC-3'; CD44-R, 5'GATGCCAAGATGATCAGCCATTCTGGAAT-3'; ALDH1A1-F 5'-GCACGCCAGACTTACCTGTC-3'; ALDH1A1-R 5'-CCTCCTCAGTTGCAGGATTAAAG3'; Oct4-F 5'-TGGGATATACACAGGCCGATG-3'; Oct4-R 5'-TCCTCCACCCACTTCTGAG-3'; Nanog-F 5'-TTTGTGGGCCTGAAGAAAACT-3'; Nanog-R 5'AGGGCTGTCCTGAATAAGCAG-3'; GAPDH-F 5' CAAGGTCACCATGACAACTTTG-3';

\section{GAPDH-R 5'-GTCCACCACCCTGTTGCTGTAG-3'}

\section{Detection of CD133 positive cells by flow cytometry}

786-O and ACHN adherent cells and tumorpheres were treated with selective concentrations of CSE for 5 days respectively, and cells were collected and washed by icecold PBS twice. Afterwards, $1 \times 10^{6}$ cells were exposed to $1 \mu \mathrm{L}$ APC-conjugated human monoclonal CD133/1 (AC133) (Miltenyi Biotech, Teterow, Germany) antibody or isotype control antibody (Mouse IgG1) (Miltenyi Biotech) at $4{ }^{\circ} \mathrm{C}$ in the darkness for 10 min subsequently resuspended in $400 \mu \mathrm{l}$ PBS and detected by flow cytometry analysis.

\section{Cell proliferation assay}

Cells grew in SFM at a density of $1 \times 10^{3}$ cells/well in a 96-well plates were renewed half every other day. After 


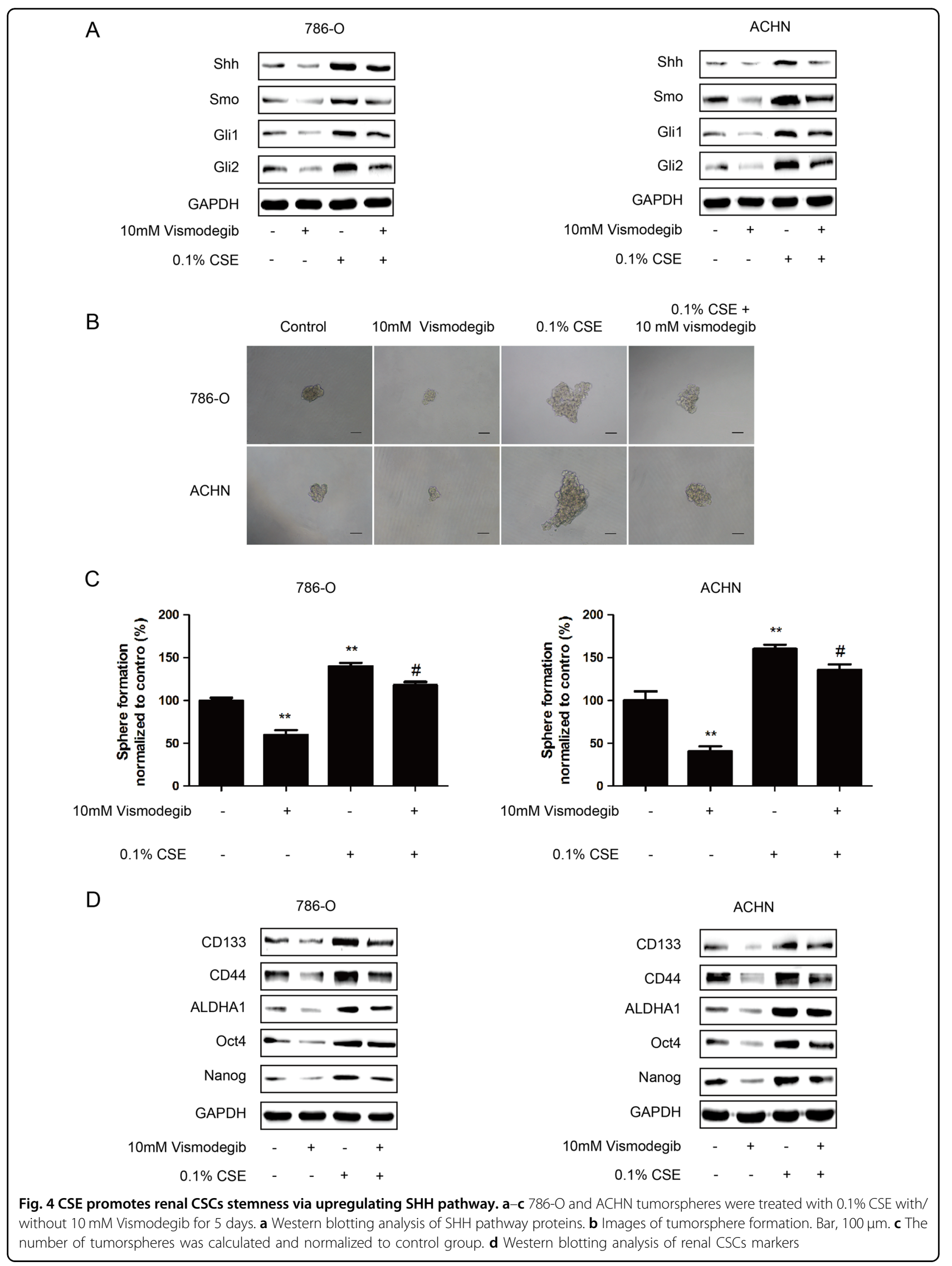




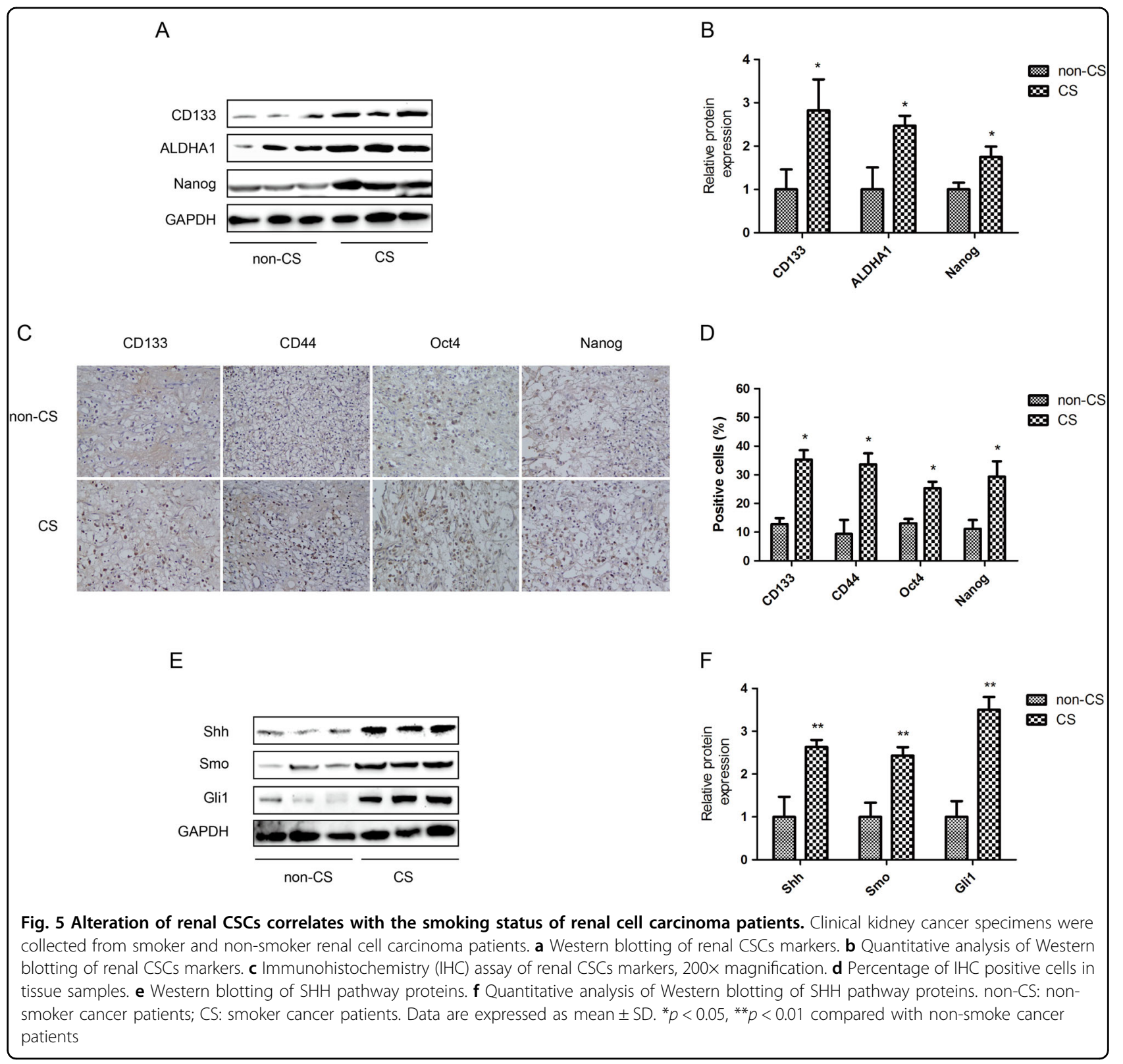

5 days, cell proliferation assay was conducted using cell counting kit 8 (CCK-8) according to the manufacturer's instructions. After incubating at $37^{\circ} \mathrm{C}$ for $3 \mathrm{~h}$, cell viability was measured at $450 \mathrm{~nm}$ absorbance by a microplate reader (Titertek Instruments Inc, USA). Each experiment was performed in triplicates.

\section{Immunofluorescence staining}

786-O and ACHN cells were seeded into 6-well culture plates at a density of $5 \times 10^{4}$ per well. After 5 days, cells were fixed, washed, and then incubated with CD44 (1:200) antibodies at $4{ }^{\circ} \mathrm{C}$ overnight. Next day, cells were stained with Cy3-conjugated goat anti-rabbit secondary for $2 \mathrm{~h}$. After washing three times, cells were incubated with DAPI for $15 \mathrm{~min}$. Images were obtained by a reversed fluorescence microscopy (Nikon, Japan).

\section{Patients and tumor specimens}

A total of 20 renal cell carcinoma patients were not treated with anti-tumor regimens before undergoing surgical operation in the Second Affiliated Hospital of Anhui Medical University, and their postoperational specimens were obtained. All the tissue sections were evaluated by experienced pathologists to confirm the 
diagnosis of renal cell carcinoma with WHO classification. The present study was approved by the Ethics Committee of Anhui Medical University. Written informed consent was obtained from the patients for this study.

\section{Immunohistochemistry (IHC)}

IHC was used to analyze the expression of renal CSCs markers (CD133, CD44, Oct4, and Nanog) in samples of postoperative tumor tissues. The fixed specimens were paraffin-embedded and cut into $4 \mu \mathrm{m}$ slides. Slides were then deparaffinized in xylene, hydrated in a graded ethanol series, and subjected to antigen retrieval with citrate buffer. After blocking with serum at room temperature, sections were incubated with primary antibodies at $4{ }^{\circ} \mathrm{C}$ overnight and incubated with secondary antibodies next day. Afterwards, the sections were briefly counterstained with diaminobenzidine (DAB) for the chromogenic reaction, and the nuclei were stained with hematoxylin. Eventually, slides were observed under an optical microscope (Nikon, Japan).

\section{Statistical analysis}

All experiments were repeated at least three times and representative results are presented. Data are expressed as the mean $\pm \mathrm{SD}$. Statistical significance $\left(" p<0.05,{ }^{* *} p<\right.$ 0.01 ) was determined by two-tailed student $t$-test between two groups and One-way ANOVA analyses of variance among groups by SPSS 16.0.

\section{Acknowledgements}

This work was supported by grants from the National Natural Science Foundation of China (No. 81373005) and Anhui Medical University scientific research funds (Hefei, China; grant No. H0514) and Anhui public welfare research linkage plan (No. 15011d04045)

\section{Author details}

'Department of Urology, The Second Affiliated Hospital of Anhui Medical University, Hefei 230032, China. ${ }^{2}$ Department of Nutrition and Food Safety, School of Public Health, Nanjing Medical University, Nanjing 211166, China. ${ }^{3}$ Department of Oncology, The First Affiliated Hospital of Anhui Medica University, Hefei 230032, China

\section{Conflict of interest}

The authors declare that they have no conflict of interest.

\section{Publisher's note}

Springer Nature remains neutral with regard to jurisdictional claims in published maps and institutional affiliations.

Received: 30 July 2017 Accepted: 5 December 2017

Published online: 13 March 2018

\section{References}

1. Siegel, R. L. et al. Cancer statistics for Hispanics/Latinos, 2015. CA 65, 457-480 (2015).

2. Jonasch, E., Gao, J. \& Rathmell, W. K. Renal cell carcinoma. Urologe 50, 219 (2011).
3. Siddique, H. R. \& Saleem, M. Role of BMI1, a stem cell factor, in cancer recurrence and chemoresistance: preclinical and clinical evidences. Stem Cells 30, 372 (2012).

4. Mummery, C., Wilmut, S. I., Stolpe, A. V. D. \& Roelen, B. A. J. Stem Cells in Cancer and Cancer Stem Cells 237-256 (Elsevier, 2011).

5. Jordan, C. T., Guzman, M. L. \& Noble, M. Cancer stem cells. N. Engl. J. Med. $\mathbf{3 5 5}$ 1253 (2006)

6. Ajani, J. A., Song, S., Hochster, H. S. \& Steinberg, I. B. Cancer stem cells: the promise and the potential. Semin. Oncol. 42, S3 (2015). Suppl 1.

7. Vlashi, E. \& Pajonk, F. Cancer stem cells, cancer cell plasticity and radiation therapy. Semin. Cancer Biol. 31, 28-35 (2015).

8. Vaiopoulos, A. G., Kostakis, I. D., Koutsilieris, M. \& Papavassiliou, A. G. Colorectal cancer stem cells. Stem Cells 30, 363-371 (2012).

9. Lichner, Z. et al. miR-17 inhibition enhances the formation of kidney cancer spheres with stem cell/ tumor initiating cell properties. Oncotarget 6 5567-5581 (2015)

10. Myszczyszyn, A. et al. The role of hypoxia and cancer stem cells in renal cell carcinoma pathogenesis. Stem. Cell. Rev. 11, 919-943 (2015).

11. Ruiz, I. A. A. Hedgehog signaling and the Gli code in stem cells, cancer, and metastases. Sci. Signal. 4, pt9 (2011).

12. Maugeri-Sacca, M., Zeuner, A. \& De Maria, R. Therapeutic targeting of cancer stem cells. Front. Oncol. 1, 10 (2011)

13. Muller, J. M., Chevrier, L., Cochaud, S., Meunier, A. C. \& Chadeneau, C. Hedgehog, Notch and Wnt developmental pathways as targets for anticancer drugs. Drug. Discov. Today Dis. Mech. 4, 285-291 (2007).

14. Jiang, J. \& Hui, C. C. Hedgehog signaling in development and cancer. Dev. Cell. 15, 801 (2008)

15. Altaba, A. R. I., Mas, C. \& Stecca, B. The Gli code: an information nexus regulating cell fate, stemness and cancer. Trends Cell. Biol. 17, 438 (2007).

16. Znaor, A., Lortettieulent, J., Laversanne, M., Jemal, A. \& Bray, F. International variations and trends in renal cell carcinoma incidence and mortality. Eur. Urol. 67, 519 (2015)

17. An, $Y$. et al. Cigarette smoke promotes drug resistance and expansion of cancer stem cell-like side population. PLOS. ONE. 7, e47919 (2012).

18. Dawood, S., Austin, L. \& Cristofanilli, M. Cancer stem cells: implications for cancer therapy. Oncology 28, 1101 (2014).

19. Valentina, P. et al. Identification and characterization of cancer stem cells from head and neck squamous cell carcinoma cell lines. Cell. Physiol. Biochem. 36, 784-798 (2015).

20. House, C. D., Hernandez, L. \& Annunziata, C. M. In vitro enrichment of ovarian cancer tumor-initiating cells. J. Vis. Exp. 96, e52446-e (2015).

21. Sun, C. et al. CD133 expression in renal cell carcinoma (RCC) is correlated with nuclear hypoxia-inducing factor 1alpha (HIF-1alpha). J. Cancer Res. Clin. Oncol. 138, 1619-1624 (2012)

22. Wu, Y. \& Wu, P. Y. CD133 as a marker for cancer stem cells: progresses and concerns. Stem. Cells Dev. 18, 1127-1134 (2009).

23. Hiraga, T., Ito, S. \& Nakamura, H. Cancer stem-like cell marker CD44 promotes bone metastases by enhancing tumorigenicity, cell motility, and hyaluronan production. Cancer Res. 73, 4112-4122 (2013).

24. Xu, X. et al. Aldehyde dehydrogenases and cancer stem cells. Cancer Lett. 369 50-57 (2015).

25. Khan, M. I. et al. Current approaches in identification and isolation of human renal cell carcinoma cancer stem cells. Stem Cell Res Ther. 6, 178 (2015).

26. Hofmann, J. N. et al. Risk of kidney cancer and chronic kidney disease in relation to hepatitis $C$ virus infection: a nationwide register-based cohort study in Sweden. Eur. J. Cancer Prev. 20, 326 (2011).

27. Kroeger, N. et al. Smoking negatively impacts renal cell carcinoma overall and cancer-specific survival. Cancer 118, 1795-1802 (2012).

28. Liu, Y. et al. Epithelial-mesenchymal transition and cancer stem cells, mediated by a long non-coding RNA, HOTAIR, are involved in cell malignant transformation induced by cigarette smoke extract. Toxicol. Appl. Pharmacol. 282, 9-19 (2015)

29. Shigemura, K. \& Fujisawa, M. Hedgehog signaling and urological cancers. Curr. Drug. Targets 16, 258-271 (2015).

30. Heiden, $\mathrm{K}$. et al. The sonic hedgehog signaling pathway maintains the cancer stem cell self-renewal of anaplastic thyroid cancer by inducing snail expression. J. Clin. Endocrinol. Metab. 99, E2178-E2187 (2014).

31. Fu, J. et al. GANT-61 inhibits pancreatic cancer stem cell growth in vitro and in NOD/SCID/IL2R gamma null mice xenograft. Cancer Lett. 330, 22-32 (2013). 
32. Dormoy, $\mathrm{V}$. et al. The sonic hedgehog signaling pathway is reactivated in human renal cell carcinoma and plays orchestral role in tumor growth. Mol. Cancer 8, 123 (2009).

33. Dormoy, V. et al. Vitamin D3 triggers antitumor activity through targeting hedgehog signaling in human renal cell carcinoma. Carcinogenesis 33, 2084-2093 (2012).
34. Liang, Z. et al. Curcumin reversed chronic tobacco smoke exposure induced urocystic EMT and acquisition of cancer stem cells properties via Wnt/ $\beta$ catenin. Cell Death Dis 8, e3066 (2017).

35. Hirata, N., Yamada, S., Sekino, Y. \& Kanda, Y. Tobacco nitrosamine NNK increases ALDH-positive cells via ROS-Wnt signaling pathway in A549 human lung cancer cells. J. Toxicol. Sci. 42, 193-204 (2017). 